Research Paper

\title{
Differential expression and clinical significance of epithelial-mesenchymal transition markers among different histological types of triple-negative breast
}

\section{cancer}

\author{
Shuling Zhou ${ }^{1,2}$, Xiangjie Sun ${ }^{1,2}$, Lin Yu ${ }^{1,2}$, Ruoji Zhou ${ }^{1,2}$, Anqi Li1 ${ }^{1,2}$, Ming Li ${ }^{1,2}$, Wentao Yang ${ }^{1,2} \bowtie$ \\ 1. Department of Pathology, Fudan University Shanghai Cancer Center, Shanghai, P.R. China; \\ 2. Department of Oncology, Shanghai Medical College, Fudan University, Shanghai, P.R. China
}

$\triangle$ Corresponding author: Wen-Tao Yang, M.D. \& Ph.D, Department of Pathology, Fudan University Shanghai Cancer Center, Department of Oncology, Shanghai Medical College, Fudan University, 270 Dong' an Road, Shanghai, 200032, P.R. China. Fax: +86-21- 64031696; Email: yangwt2000@163.com

(c) Ivyspring International Publisher. This is an open access article distributed under the terms of the Creative Commons Attribution (CC BY-NC) license (https://creativecommons.org/licenses/by-nc/4.0/). See http://ivyspring.com/terms for full terms and conditions.

Received: 2017.01.15; Accepted: 2017.08.20; Published: 2018.01.05

\begin{abstract}
Background: Triple-negative breast cancer (TNBC) is a heterogeneous disease closely associated with epithelial-to-mesenchymal transition (EMT). This study aimed to investigate the role of EMT in metaplastic carcinoma.

Methods: E-cadherin, Slug, Twist and Vimentin levels were detected by immunohistochemistry in 167 TNBC tumors, including 145 invasive carcinomas of no special type (ICONSTs), 14 spindle cell carcinomas (SpCCs) and 8 matrix-producing carcinomas (MPCs).

Results: Nuclear Slug and Twist were more frequently detected in SpCC and MPC tumors than that in ICONST tumors $(p<0.001)$. The rate of E-cadherin loss was much lower in the ICONST tumors than that in the SpCC and MPC tumors $(p<0.001)$. Vimentin was expressed in all SpCC and MPC tumors. Furthermore, nuclear expression of Slug and Twist was positively associated with the cytoplasmic localization of Vimentin $(p<0.001)$ and was inversely associated with membranous staining of E-cadherin $(p<0.001)$. These trends were more apparent in the SpCC and MPC tumors than in the ICONST tumors. Follow-up data were available for 151 patients. The follow-up times ranged from 1 month to 11 years (mean: $74 \mathrm{~m}$; median: $21 \mathrm{~m}$ ). The median progression-free survival and overall survival times were 24 months (mean: 32 months) and 22 months (mean: 35 months), respectively. Tumor size, TNM stage and E-cadherin were found to be independent prognostic factors of TNBC.

Conclusions: EMT may play an important role in TNBC, especially in MPC and SpCC. Further researches are needed to confirm this finding. The results of this study may facilitate the future development of targeted therapies based on alterations in the EMT and stem cell markers.
\end{abstract}

Key words: epithelial-mesenchymal transition, breast cancer, triple negative, metaplastic carcinoma, clinical significance

\section{Introduction}

Microarray-based gene expression profiling studies performed over the past decade have identified four main intrinsic molecular subtypes of breast cancer, including the luminal A, luminal B, human epidermal growth factor receptor 2 (HER2)-enriched and basal-like subtypes [1-4]. Triple-negative breast cancer (TNBC) is defined by the absence of detectable estrogen receptor (ER) and progesterone receptor (PR) expression and the lack of HER2 gene amplification. The features of the TNBC roughly parallel those of the basal-like subtype. Emerging data clearly indicate that TNBC is a heterogeneous disease with a variable prognosis that depends on clinical, pathologic, and genetic factors. Recently, Lehmann et al. [5] have found that TNBC can be classified into 7 subtypes (6 defined subtypes 
and an unstable group) by gene expression microarray analysis. The 7 TNBC subtypes were characterized on the basis of gene ontologies and differential gene expression and were labeled as basal-like 1 (BL1), basal-like 2 (BL2), immunomodulatory (IM), mesenchymal (M), mesenchymal stem-like (MSL), luminal androgen receptor (LAR), and unstable (UNS). Both the M and MSL subtypes are characterized by the predominant expression of genes involved in motility, the extracellular matrix, and cell differentiation pathways, as well as genes associated with the epithelial-to-mesenchymal transition (EMT). The EMT is important in embryogenesis, tissue regeneration, inflammation, wound healing, fibrosis, and carcinogenesis [6]. These processes are associated with down-regulation of the expression of epithelial cell junction proteins, such as E-cadherin, and up-regulation of the expression of mesenchymal cytoskeletal proteins, such as Vimentin. Interestingly, the M and MSL subtypes of TNBC have been closely linked to metaplastic breast cancer.

Slug and Twist, which are known to promote EMT during development, have been reported to be preferentially expressed in TNBC but not in the luminal A/B or HER2-positive subtypes [7,8]. The expression levels of EMT-related genes (CDH1, Twist, and SNAIL1) in invasive carcinoma of no special type (ICONST) and their correlations with patient prognosis have been reported [7]; however, the role of the EMT in the other histologic subtypes of breast cancer remain to be elucidated. Here, we hypothesized that the expression of EMT markers would be more frequent in metaplastic carcinoma than that of ICONST. Thus, to further clarify this issue, we assessed the expression of EMT markers (E-cadherin, Vimentin, Slug and Twist) in a series of well-characterized patients with TNBC, including patients with metaplastic breast cancer and ICONST. We aimed to further examine the correlations between the histological subtypes and the molecular subtypes of triple-negative (TN) tumors.

\section{Materials and Methods}

\section{Case selection}

A total of 167 patients who underwent surgery at Fudan University, Shanghai Cancer Center from February 1, 2001 to December 31, 2012 were consecutively retrieved from a pathology database. The patients were recruited according to the following criteria: (i) histologically confirmed ICONST/spindle cell carcinoma (SpCC)/matrix-producing carcinoma (MPC) with an ER-/PR-/HER2-negative phenotype; (ii) no evidence of distant metastasis at diagnosis; (iii) no type of treatment prior to surgery. Finally, 145 patients with ICNOST, 14 with SpCC and 8 with MPC were included. All 167 patients had TN tumors. The ER, PR and HER2 statuses were evaluated according to the scoring criteria of the American Society of Clinical Oncology/College of American Pathologists $[9,10]$. Tumor stage (TNM) was evaluated according to the seventh edition of the American Joint Committee on Cancer (AJCC) staging system [11].

The surgical specimens were routinely processed, sectioned into $4-\mu \mathrm{m}$ sections and stained with hematoxylin and eosin (H\&E). Histological type was characterized based on the tumor classifications defined by the World Health Organization (WHO; 2012 version) [11].In addition, the tumors were graded using the Elston-Ellis histologic grading system [12].

\section{Clinicopathological parameters}

The major characteristics of TNBC patients classified by histologic subtypes are reported in Table 1. All of the patients were female, with a mean age at diagnosis of 53 years (age range: 27 to $87 \mathrm{y}$ ). The patients with ICONST were younger $(51.0 \%)$ than those with other histologic subtypes (SpCC, 14.3\% and MPC, 37.5\%), with mean ages of 51, 60 and 53 years, respectively. The majority of the SpCC patients $(92.9 \%)$ were postmenopausal, and approximately half of the ICONST $(54.5 \%)$ and MPC $(50 \%)$ patients were postmenopausal. All of the metaplastic tumors were classified as high grade. The maximum tumor diameters varied among the different subtypes, with mean of $2.9 \mathrm{~cm}$. SpCC $(35.7 \%)$ and MPC $(25 \%)$ tumors were more frequently larger in size $(>5 \mathrm{~cm})$ than ICONST tumors (4.1\%). Positive lymph node involvement was reported for $42.0 \%$ of the ICONST patients, $16.7 \%$ of the SpCC patients, and $37.5 \%$ of the MPC patients.

Significant differences in several characteristics, including age, menopausal status, and tumor grade and size, were observed among the three histological types (Table 1). Therefore, we conducted a further pairwise comparison to assess the differences among the three groups in detail. Most of the SpCC patients were postmenopausal and had an older age $(p=0.009)$, a higher histological grade $(p=0.006)$, and a larger tumor size $(\mathrm{p}<0.001)$ compared with the ICONST patients. A large tumor size $(>5 \mathrm{~cm})$ and high histological grade are more commonly observed in MPC than in ICONST. The tumor stage appeared to be higher (stage III) for the MPC patients than for the ICONST patients; however, this difference was not significant. 
Table 1. Clinicopathological characteristics of different histological subtypes

\begin{tabular}{|c|c|c|c|c|c|c|c|c|}
\hline Characteristics & $\begin{array}{l}\text { Total } \\
N(\%) \\
N=167\end{array}$ & $\begin{array}{l}\text { ICONST } \\
\mathrm{N}(\%) \\
\mathrm{N}=145\end{array}$ & $\begin{array}{l}\text { SpCC } \\
N(\%) \\
N=14\end{array}$ & $\begin{array}{l}\mathrm{MPC} \\
\mathrm{N}(\%) \\
\mathrm{N}=8\end{array}$ & $\begin{array}{l}\text { p-value } \\
\text { three subtypes }\end{array}$ & $\begin{array}{l}\text { p-value } \\
\text { ICONST vs. SpCC }\end{array}$ & $\begin{array}{l}\mathrm{p} \text {-value } \\
\text { ICONST vs. MPC }\end{array}$ & $\begin{array}{l}\text { p-value } \\
\text { SpCC vs. MPC }\end{array}$ \\
\hline Age (mean 53 y, range $27-87$ y) & & & & & 0.027 & 0.009 & 0.4 & 0.2 \\
\hline$\leq 50 \mathrm{y}$ & $79(47.3)$ & $74(51.0)$ & $2(14.3)$ & $3(37.5)$ & & & & \\
\hline$>50 y$ & $88(52.7)$ & $71(49.0)$ & $12(85.7)$ & $5(62.5)$ & & & & \\
\hline Menopausal status & & & & & 0.021 & 0.006 & 0.7 & 0.02 \\
\hline Premenopausal & $71(42.5)$ & $66(45.5)$ & $1(7.1)$ & $4(50.0)$ & & & & \\
\hline Postmenopausal & $96(57.5)$ & $79(54.5)$ & 13(92.9) & $4(50.0)$ & & & & \\
\hline Grade & & & & & $<0.001$ & $<0.001$ & $<0.001$ & / \\
\hline I & / & / & / & / & & & & \\
\hline II & $103(61.7)$ & 103(71.0) & / & / & & & & \\
\hline III & $64(38.3)$ & $42(29.0)$ & $14(100)$ & $8(100)$ & & & & \\
\hline $\begin{array}{l}\text { Size } \\
(\text { mean } 2.9 \mathrm{~cm} \text {, range } 0.5-14 \mathrm{~cm})\end{array}$ & & & & & $<0.001$ & $<0.001$ & 0.02 & 0.4 \\
\hline$\leq 2$ & $61(36.5)$ & $58(40.0)$ & $1(7.1)$ & $2(25.0)$ & & & & \\
\hline $2-5$ & $93(55.7)$ & $81(55.9)$ & $8(57.1)$ & $4(50.0)$ & & & & \\
\hline$>5$ & $13(7.8)$ & $6(4.1)$ & $5(35.7)$ & $2(25.0)$ & & & & \\
\hline Lymph node status & & & & & 0.2 & 0.09 & 0.8 & 0.2 \\
\hline Positive & $66(40.0)$ & $61(42.0)$ & $2(16.7)$ & $3(37.5)$ & & & & \\
\hline Negative & $99(60.0)$ & $84(58.3)$ & $10(83.3)$ & $5(62.5)$ & & & & \\
\hline Not evaluated & 2 & / & 2 & / & & & & \\
\hline TNM stage ${ }^{a}$ & & & & & 0.4 & 0.2 & 0.9 & 0.3 \\
\hline I & $39(23.6)$ & $36(24.8)$ & $1(8.3)$ & $2(25.0)$ & & & & \\
\hline II & $91(55.1)$ & $77(53.1)$ & $10(83.3)$ & $4(50.0)$ & & & & \\
\hline III & $35(21.2)$ & $32(22.1)$ & $1(8.3)$ & $2(25.0)$ & & & & \\
\hline
\end{tabular}

a. Since the lymph node status of two patients was not evaluated, TNM stage of them was not assessed.

\section{Patient follow-up}

We obtained clinical data on the surgical procedure used, survival status after surgery, local recurrence (including residual breast tissue and the chest wall) and distant metastasis. Metastatic sites were detected clinically or by imaging. The survival data included progression-free survival (PFS) and overall survival (OS). The former was defined as the time from the date of surgery to any type of recurrence, distant metastasis, or death from breast cancer or to the last follow-up, and the latter was defined as the time from surgery to the date of death from breast cancer or to the last follow-up.

\section{Tissue microarrays}

Three tissue microarrays were used in this study. A total of $150 \mathrm{TN}$ ICONST breast cancer tissues collected from 2001 to 2009 were included in these arrays. The representative tumor areas were selected using matched H\&E-stained slides and marked directly on the donor block. Each cylindrical tissue sample was cored (diameter of $2 \mathrm{~mm}$ ) within the selected region of the donor block and transferred directly to the recipient block. The percentage of tissue core that contained tumor tissue was $>70 \%$. All tissue samples included in the present study were double-pseudomized leftover samples that had been used for routine pathological diagnoses; these samples could be used for research purposes without obtaining informed consent. Control tissues were acquired by obtaining normal breast tissues from the cancer patients. Multiple $4-\mu \mathrm{m}$ sections were cut with a microtome and transferred to slides.

\section{Immunohistochemical analysis of Slug, Twist, E-cadherin, and Vimentin}

Twenty-two samples of tumors with specific histological types of TNBC were collected from the patients between 2005 and 2012, routinely processed and sectioned into $4-\mu \mathrm{m}$ sections. Antibody staining for Slug, Twist, E-cadherin, and Vimentin was performed manually using an EnVision ${ }^{\mathrm{TM}}$ Detection System (DAKO). Details on the primary antibodies, dilutions, antigen retrieval methods, and suppliers are listed in Table 2. Both positive and negative controls were used throughout the analysis. A two-tiered (negative vs. positive) scoring system was used. E-cadherin protein expression was localized to the membrane. Cytoplasmic staining was considered positive for Vimentin. For the transcription factors Slug and Twist, detectable immunoreactivity in the nuclear region was defined as positive expression. Tumors were regarded as immunopositive if $>10 \%$ of tumor cells showed immunoreactivity $[7,13]$.

\section{Statistical analysis}

All statistical analyses were performed using SPSS 20.0 software (SPSS Inc., Chicago, IL). Between-group comparisons were performed using the chi-square test and t-test. OS and PFS were 
calculated using the Kaplan-Meier estimator and validated using the log-rank test. Correlation analysis was performed with Spearman's rank test. All statistical tests were two-sided, and differences were considered significant at a $p$ value of $<0.05$.

Table 2. Antibodies used in this study for immunohistochemical analysis of TNBC

\begin{tabular}{lllll}
\hline Antibody & Clone & Dilution & Supplier & $\begin{array}{l}\text { Antigen Retrieval } \\
\text { Solution }\end{array}$ \\
\hline ER & SP1 & No dilution & Ventana & EDTA (pH 9.0) \\
PR & 1E2 & No dilution & Ventana & EDTA (pH 9.0) \\
HER2 & 4B5 & No dilution & Ventana & EDTA (pH 9.0) \\
E-cadherin & MAB-0 & No dilution & MXB & Citrate buffer (pH 6.0) \\
& 589 & & Biotechnologies & \\
Vimentin & MAB-0 & No dilution & MXB & Citrate buffer (pH 6.0) \\
& 178 & & Biotechnologies & \\
Twist & ab50887 & $1: 200$ & Abcam & EDTA (pH 9.0) \\
Slug & C19G7 & $1: 100$ & Cell Signaling & EDTA (pH 9.0) \\
& & & Technology & \\
\hline
\end{tabular}

\section{Results}

\section{Immunohistochemical analysis of E-cadherin, Vimentin, Slug and Twist in the three histological subtypes of TNBC}

The results of immunohistochemical analyses of the 167 patients' tumor samples are shown in Table 3, and representative immunohistochemical staining images are shown in Fig. 1. Of the 145 ICONST tumors examined, 31/145 (21.4\%) showed loss of membranous staining for E-cadherin in tumor cells, while only 1 SpCC tumor showed positive membranous staining for E-cadherin. None of the MPC tumors expressed E-cadherin. A significant inverse association was observed between membranous staining for E-cadherin and histological grade $(\mathrm{p}<0.001)$ (Supplemental Table 1$)$.

Slug and Twist were expressed in $28(19.3 \%)$ and $59(40.7 \%)$ of the 145 ICONST tumors examined (Fig. 1d, 1e), respectively. Among the 14 tumor samples from the patients with SpCC, 78.6\% expressed Slug, and $92.9 \%$ expressed Twist. All 8 MPC tumors expressed Slug and Twist. Significant positive associations were observed between nuclear accumulation of Slug and histological grade and tumor size $(\mathrm{p}=0.01)$ (Supplemental Table 1).

Vimentin expression was localized to the cytoplasm in tumor cells. A total of $44(30.3 \%)$ of the 145 ICONST tumors expressed Vimentin (Fig. 1c), and all of the SpCC and MPC tumors expressed this protein (Fig. 1h, 1m). Cytoplasmic Vimentin expression was positively associated with histological grade $(p=0.007)$ (Supplemental Table 1$)$.

\section{Slug, Twist, and Vimentin expression is increased in metaplastic subtypes}

Absent or attenuated expression of the epithelial marker E-cadherin was more common in SpCC and MPC than in ICONST $(p<0.001, p=0.04)$. Aberrant expression of the mesenchymal marker Vimentin and the EMT transcription factors Slug and Twist were observed in the SpCC and MPC tumors $(\mathrm{p}<0.001)$. The differences in Slug, Twist and Vimentin expression between SpCC and MPC were not significant (Table 3).

\section{Associations of E-cadherin and Vimentin expression with Slug and Twist expression}

Statistical analysis revealed significant associations between the EMT markers in all 167 tumors (Supplemental Table 2). Significant inverse associations were observed between nuclear expression of Slug and Twist and membranous immunostaining for E-cadherin in TNBC (Fig. 2b, 2d). In addition, positive associations were detected between the cytoplasmic Vimentin and nuclear Slug and Twist expression (Fig. 2a, 2c). These trends were more pronounced in the SpCC and MPC tumors than in the ICONST tumors (Table 3).

Table 3. Comparison of EMT marker expression patterns according to histological subtypes

\begin{tabular}{|c|c|c|c|c|c|c|c|c|}
\hline & Total & ICONST & SpCC & MPC & $\mathrm{p}$-value & $\mathrm{p}$-value & $\mathrm{p}$-value & $\mathrm{p}$-value \\
\hline & $\mathrm{N}(\%)$ & $\mathrm{N}(\%)$ & $\mathrm{N}(\%)$ & $\mathrm{N}(\%)$ & three subtypes & ICONST vs. SpCC & ICONST vs. MPC & SpCC vs. MPC \\
\hline & $\mathrm{N}=167$ & $\mathrm{~N}=145$ & $\mathrm{~N}=14$ & $\mathrm{~N}=8$ & & & & \\
\hline E-cadherin & & & & & $<0.001$ & $<0.001$ & 0.04 & 0.439 \\
\hline Positive & $115(68.9)$ & $114(78.6)$ & $1(7.1)$ & $0(0.0)$ & & & & \\
\hline Negative & $52(31.1)$ & $31(21.4)$ & $13(92.9)$ & $8(100.0)$ & & & & \\
\hline Slug & & & & & $<0.001$ & $<0.001$ & $<0.001$ & 0.159 \\
\hline Positive & $47(28.1)$ & 28(19.3) & 11(78.6) & $8(100.0)$ & & & & \\
\hline Negative & $120(71.9)$ & $117(80.7)$ & $3(21.4)$ & $0(0.0)$ & & & & \\
\hline Twist & & & & & $<0.001$ & $<0.001$ & $<0.001$ & 0.159 \\
\hline Positive & $80(47.9)$ & $59(40.7)$ & $13(92.9)$ & $8(100.0)$ & & & & \\
\hline Negative & $87(52.1)$ & $86(59.3)$ & $1(7.1)$ & $0(0.0)$ & & & & \\
\hline Vimentin & & & & & $<0.001$ & $<0.001$ & $<0.001$ & 0.439 \\
\hline Positive & $66(39.5)$ & $44(30.3)$ & $14(100.0)$ & $8(100.0)$ & & & & \\
\hline Negative & $101(60.5)$ & $101(69.7)$ & $0(0.0)$ & $0(0.0)$ & & & & \\
\hline
\end{tabular}



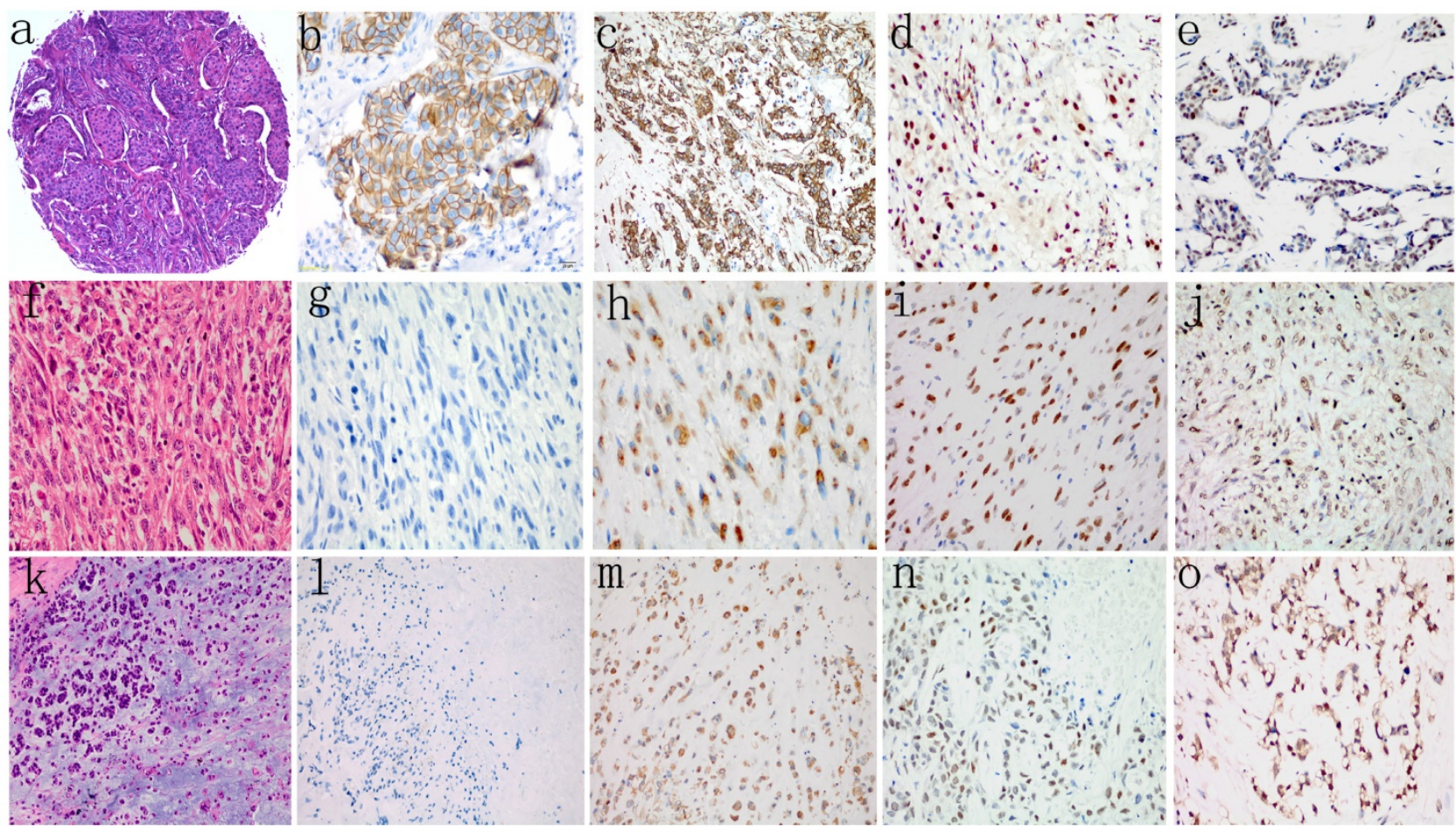

Figure 1. H\&E staining $(a, f, k)$ and representative images showing negative or positive expression of E-cadherin (b, g, I), Vimentin (c, h, m), Slug (d, i, $n$ ), and Twist $(e, j, o)$ in different histological types of TNBC. Loss of membranous staining of E-cadherin was detected in SpCC (g) and MPC (l). Cytoplasmic expression of Vimentin was observed in both SPCC (h) and MPC (m). Vimentin was also expressed in several ICONST tumors (c). Nuclear expression of Slug and Twist was frequently observed in $\operatorname{SpCC}(\mathrm{i}, \mathrm{j})$ and MPC $(\mathrm{n}, \mathrm{o})$ tumors.

a

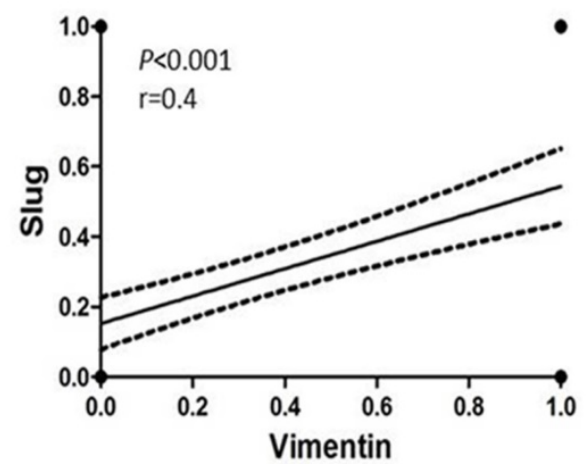

C

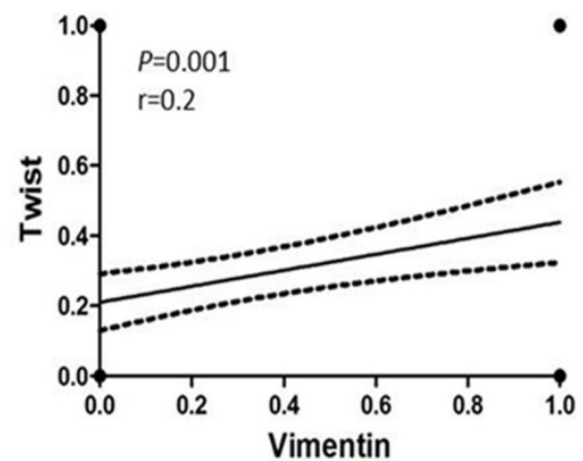

b

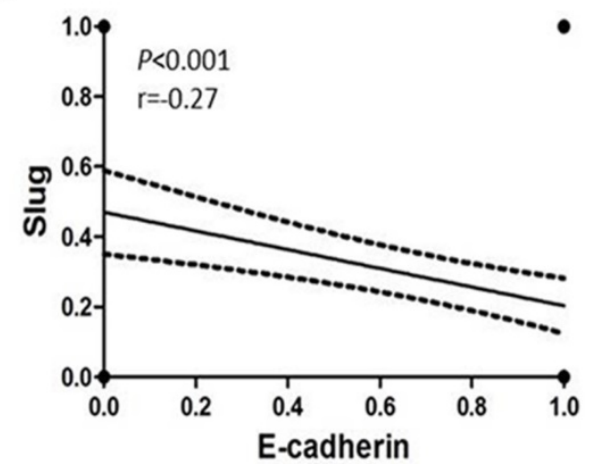

d

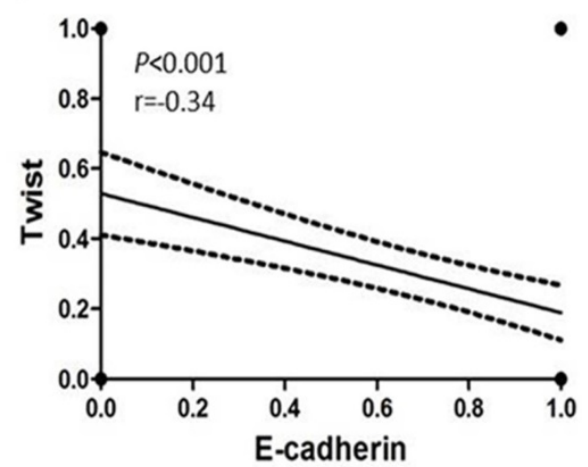

Figure 2. Relationships of E-cadherin and Vimentin expression with Slug and Twist expression. Slug and Twist expression was positively associated with Vimentin expression (a, c) and inversely associated with E-cadherin expression (b, d). 


\section{Treatment and follow up}

Modified radical mastectomy was the initial therapy in 150 of the 167 patients. Conservative surgery was performed for 8 patients ( 2 with sentinel lymph node biopsy and 7 with axillary lymph node dissection). Simple mastectomy with sentinel lymph node biopsy was performed for 9 patients.

Follow-up data were available for 151 patients. The follow-up times ranged from 1 month to 11 years, with mean and median postsurgical intervals of 74 and 21 months, respectively. Overall, disease progression (defined as recurrence, metastasis or death resulting from disease) occurred in 35 patients (21\%). The median PFS time was 24 months (mean of 32 months). Twenty-two of the 35 patients died of breast cancer by the last follow-up, with a median OS time of 22 months (mean of 35 months).

Fig. 3 shows the PFS and OS rates for each histologic subtype of TNBC. The 5-year PFS rates were $77 \%, 56.2 \%$ and $75.0 \%$ for the patients with ICONST, SpCC and MPC breast cancer, respectively. Further, the 5-year OS rates were $84.4 \%, 61.9 \%$, and $70 \%$ for the patients with ICONST, SpCC, and MPC, respectively.

\section{Survival analysis}

PFS and OS were reduced in patients with SpCC and MPC ( $p=0.27$ and $p=0.106$, respectively) compared with those in patients with ICONST, although these differences were not significant (Fig. 3). Univariate (Table 4, Fig. 4) and multivariate analyses of the associations of PFS and OS with the expression of EMT-related genes were conducted. The results revealed that tumor size, TNM stage and E-cadherin were independent prognostic factors for TNBC (Table 5).

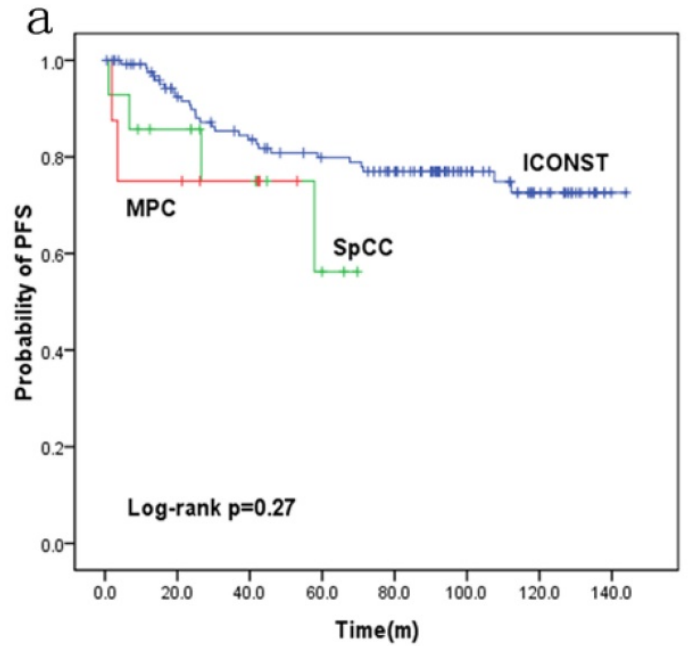

\section{Discussion}

TNBC comprises approximately $15 \%$ of all subtypes of breast cancer. Emerging evidence clearly indicates that TNBC is a heterogeneous disease with a variable prognosis that depends on clinical, pathologic, and genetic factors. Most TNBCs have been reported to be ICONST, and a number of other histological types (e.g., secretory, adenoid cystic, metaplastic, medullary, and myoepithelial tumors) can also exhibit a TNBC phenotype. Specific types of breast cancer might be associated with an extremely good or extremely poor prognosis compared with the prognosis of ICONST with similar biological features at a similar stage [12,14-17]. Metaplastic breast carcinoma comprises a heterogeneous group of tumors. In addition, some subtypes are more aggressive than ICONST, including SpCC and MPC [18-20].

SpCC of the breast presents a particular challenge, as it affects the breast but has the features of a soft tissue sarcoma. Compared with ICONST lesions, both SpCC and MPC lesions tend to be relatively large, in accordance with Gersell and Downs's findings $[18,21]$. It is important to consider the disease stage when assessing survival after treatment given that patients who present with late-stage disease will likely have poorer outcomes [20]. In our study, the five-year OS and PFS rates for the three subtypes did not significantly differ. However, we obtained different findings when we assessed the patients with early-stage disease separately from those with late-stage disease. For those with late-stage disease, the survival rates for SpCC and MPC were significantly lower than those for ICONST, although the results were based on a small number of patients. These findings are consistent with those of previous studies [20].

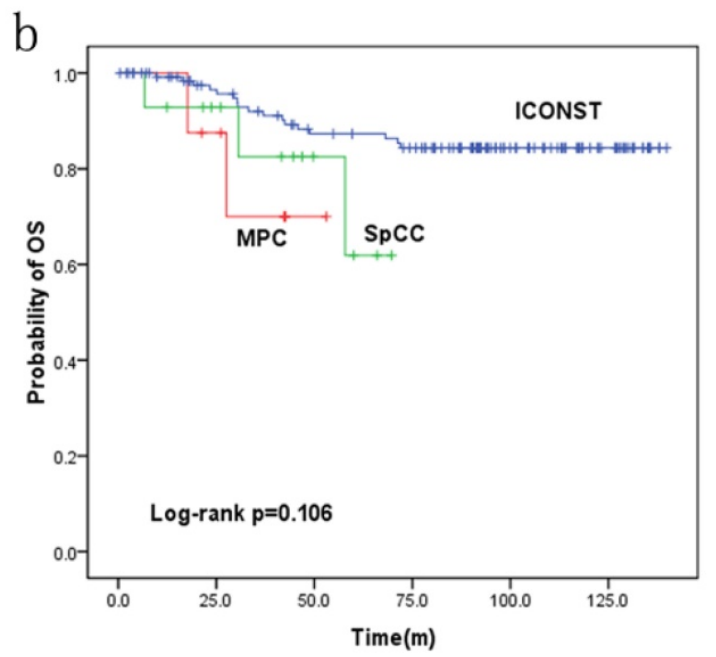

Figure 3. Progression-free survival (PFS) (a) and overall survival (OS) (b) curves for the three histological types. 
a

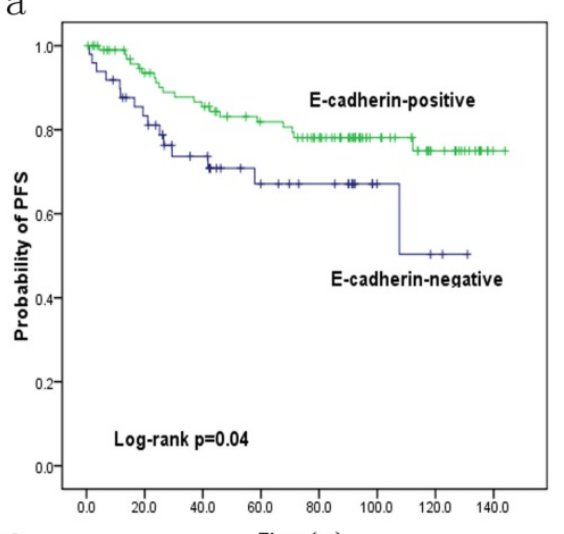

C

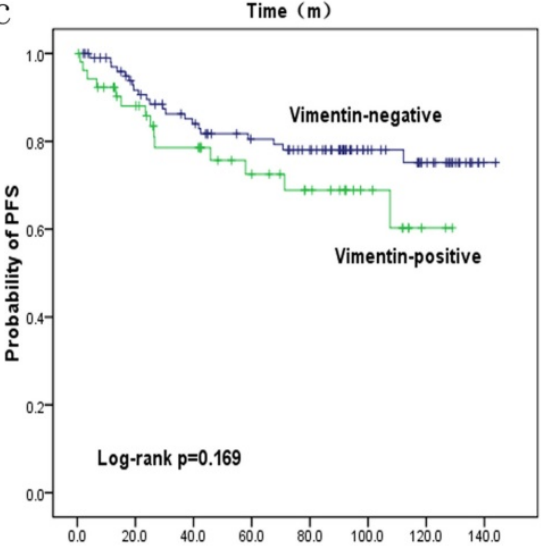

e

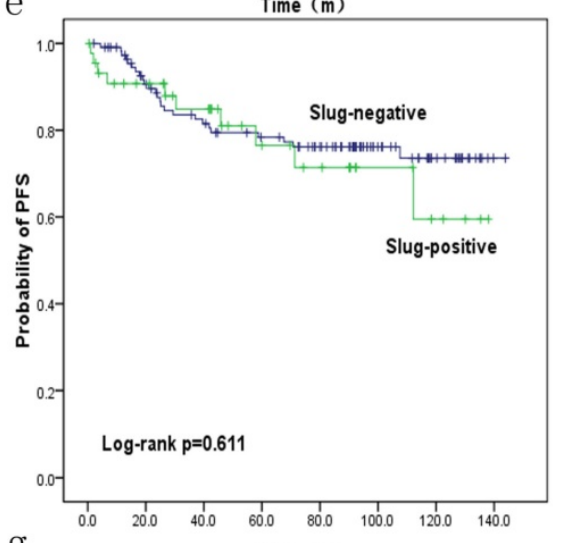

g

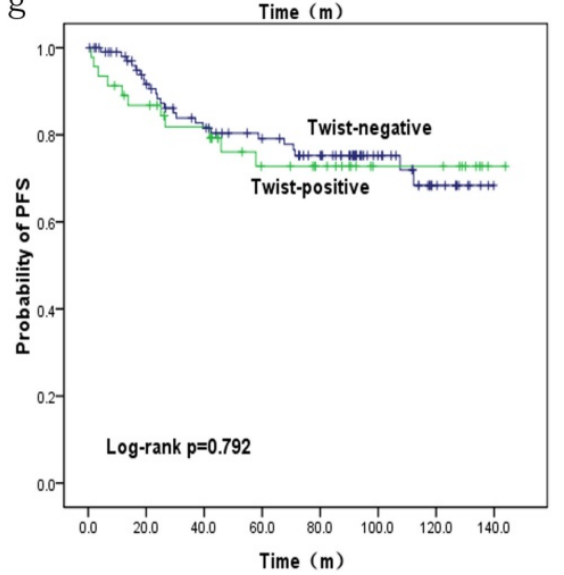

b

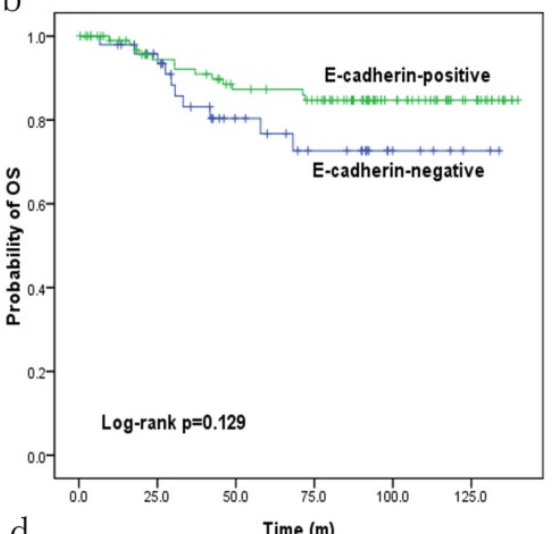

d

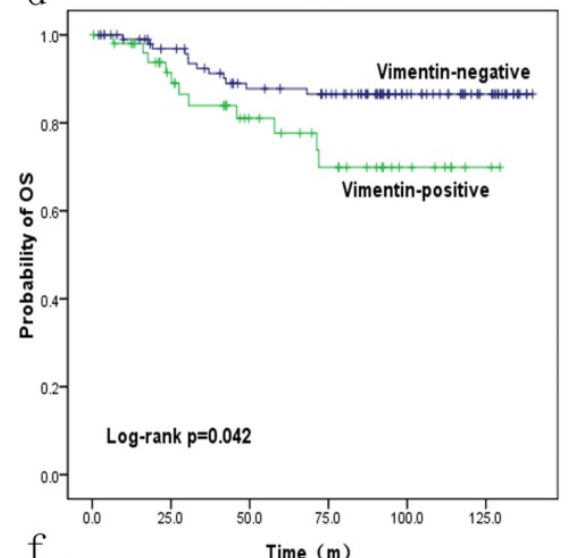

f

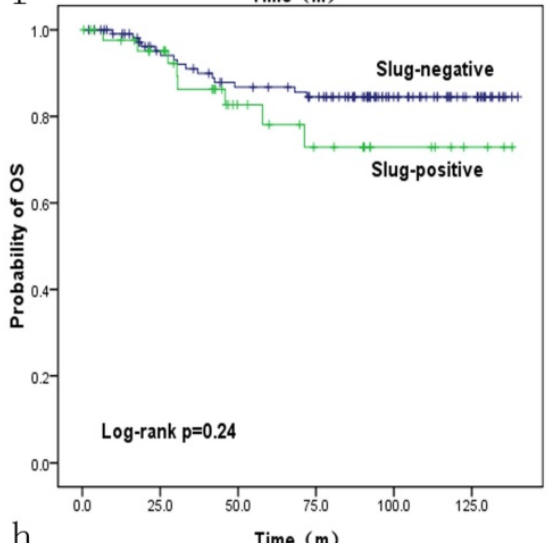

$\mathrm{h}$

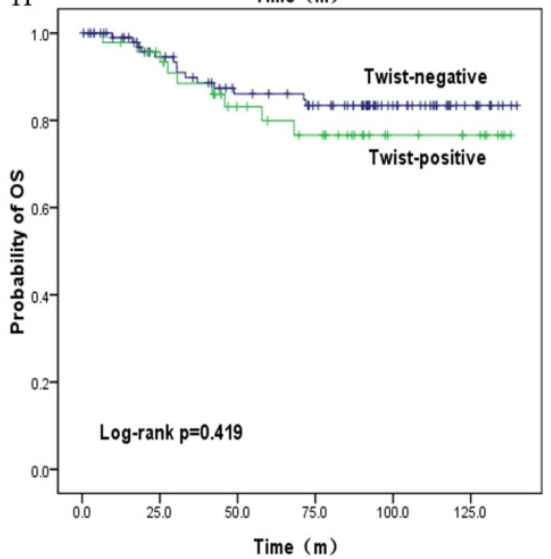

Figure 4. Overall survival (OS) (b, $d, f, h)$ and progression-free survival (PFS) (a, c, e, g) curves for patients with EMT marker expression. OS (d) and PFS (a) curves showing the poor prognosis of patients with positive Vimentin expression $(\mathrm{p}=0.042)$ and negative $\mathrm{E}$-cadherin expression $(\mathrm{p}=0.04)$, respectively. 
Table 4. Univariate regression model of prognostic covariates in TNBC patients

\begin{tabular}{llllll}
\hline & \multicolumn{2}{l}{ PFS } & & & OS \\
\cline { 2 - 3 } \cline { 5 - 6 } Biomarkers & $\chi^{2}$ & $\mathrm{p}$ & & $\chi^{2}$ & $\mathrm{p}$ \\
\hline Age & 1.231 & 0.271 & & 3.216 & 0.07 \\
Grade & 0.262 & 0.609 & & 0.84 & 0.36 \\
Size & 23.580 & $\mathbf{< 0 . 0 0 1}$ & & 44.543 & $<\mathbf{0 . 0 0 1}$ \\
TNM stage & 29.613 & $\mathbf{< 0 . 0 0 1}$ & & 41.129 & $<\mathbf{0 . 0 0 1}$ \\
E-cadherin & 4.237 & $\mathbf{0 . 0 4}$ & & 2.304 & 0.129 \\
Vimentin & 0.893 & 0.169 & & 4.151 & $\mathbf{0 . 0 4 2}$ \\
Slug & 0.259 & 0.611 & & 1.383 & 0.24 \\
Twist & 0.07 & 0.792 & & 0.654 & 0.419 \\
\hline
\end{tabular}

Table 5. Multivariate Cox regression model of TNBC patient survival

\begin{tabular}{llll}
\hline Characteristics & Outcome & $\operatorname{Exp}(\mathrm{B})(95 \% \mathrm{CI})$ & $\mathrm{p}$ \\
\hline Size & OS & $2.475(1.059-5.784)$ & $\mathbf{0 . 0 3 6}$ \\
& PFS & $1.746(0.878-3.472)$ & 0.58 \\
TNM stage & OS & $6.465(2.729-15.316)$ & $<0.001$ \\
& PFS & $3.168(1.635-6.14)$ & $\mathbf{0 . 0 0 1}$ \\
E-cadherin & OS & $0.697(0.225-2.163)$ & 0.533 \\
& PFS & $0.416(0.180-0.961)$ & $\mathbf{0 . 0 4}$ \\
\hline
\end{tabular}

On the basis of gene expression profiles, Lehmann et al. [5] defined six new TNBC subtypes: two basal-like (BL1 and BL2), two mesenchymal (M and MSL), one immunomodulatory (IM) and one luminal androgen receptor subtype (LAR). The M and MSL subtypes are heavily enriched in components and pathways involved in cell motility and exhibit high expression of EMT-related genes [5]. The EMT is a critical process that occurs during development and wound healing. In addition, it can facilitate tumor cell invasion, spread and metastasis, and therefore, it has been hypothesized to contribute to tumor progression and chemoresistance $[6,22,23]$. The EMT is characterized by up-regulation of the expression of Vimentin and E-cadherin repressor molecules (snail/SLUG/TWIST) and down-regulation of the expression of E-cadherin and other cell adhesion molecules [13,24,25]. Features of the EMT have been observed in a variety of cancers, including esophageal [26], ovarian [27], colon [28], bladder [29] and breast $[7,8,30,31]$ cancer models. Although EMT markers in TNBC had been studied in previous studies, our research was focused on the expression and clinical significance of EMT markers in special histological subtypes of TNBC, such as metaplastic breast tumor. In our study, we found that EMT markers were highly expressed in special TNBC subtypes such as SpCC and MPC.

Loss of E-cadherin occurs in $6 \%$ to $19 \%$ of ICONST breast tumors [32]. In the present study, membranous E-cadherin expression was suppressed in $31.1 \%(52 / 167)$ of the TNBC tumors. In addition, it was suppressed in $21.4 \%(31 / 145)$ of the ICONST tumors excluding the SpCC and MPC tumors, in which $92.9 \%(13 / 14)$ and $100 \%(8 / 8)$, respectively, exhibited the loss of E-cadherin expression. Loss of E-cadherin in ICONST tumors in this study is slightly higher than previously reported values [32]. Previous studies have suggested that the EMT is promoted within a specific genetic context, such as in TNBC [33-36]. Therefore, the fact that all ICONST tumors in our study were TNBC might explain our results. Obvious loss of membranous E-cadherin expression was observed in the SpCC and MPC tumors $(\mathrm{p}<0.001)$.

Vimentin is the mesenchymal marker most commonly associated with the EMT. It is conveniently abundant in connective tissue septa and in cells associated with blood vessels but is usually absent from the tumor parenchyma, and occasionally, it is observed focally in the parenchyma [37]. A previous study has reported that approximately $15 \%$ of invasive breast tumors express Vimentin [38]. In the current study, Vimentin was expressed in 30.3\% $(44 / 145)$ of the ICONST tumors and in all of the SpCC $(100 \%, 14 / 14)$ and MPC tumors $(100 \%, 8 / 8)$. A previous histological analysis of human breast carcinoma samples has revealed that Vimentin expression predominantly occurs in high-grade ductal carcinomas lacking ER [37]. All tumors were moderate- to high-grade tumors with a TN immunohistochemical phenotype. Therefore, the finding of the high level of Vimentin expression in this study is not surprising.

In the current study, nuclear accumulation of Slug was observed in $19.3 \%(28 / 145)$ of the ICONST tumors, $78.6 \%(11 / 14)$ of the SpCC tumors and $100 \%$ $(8 / 8)$ of the MPC tumors. Furthermore, nuclear accumulation of Twist was observed in $40.7 \%$ $(59 / 145)$ of the ICONST tumors, $92.9 \%(13 / 14)$ of the SpCC tumors and $100 \%(8 / 8)$ of the MPC tumors. Slug and Twist were highly expressed in the SpCC and MPC tumors. Compared to ICONST, SpCC and MPC were significantly enriched for EMT markers $(p<0.001)$. These findings are consistent with those of a previous study demonstrating that metaplastic breast cancer is enriched in EMT markers (e.g., TWIST, snail and SNAI2/SLUG) [39]. In addition, most metaplastic breast tumors are TN for ER, PR, and HER2 [40]. This TN status might contribute to the high expression levels of EMT markers in TNBC. Interestingly, $\mathrm{M}$ and MSL tumors have been closely linked to metaplastic breast cancer [5]. We speculate that the SpCC and MPC subtypes are molecularly related to the M/MSL subtype.

The results of our study also demonstrated a Slug- and Twist-mediated loss of E-cadherin and Vimentin expression in TNBC, especially in 
metaplastic cancer. Slug has been suggested to be an in vivo repressor of E-cadherin rather than snail in breast carcinoma [24,41]. Twist expression results in loss of E-cadherin-mediated cell-cell adhesion and activation of mesenchymal markers [42]. Furthermore, Vimentin expression is induced by Slug and is required for Slug-induced EMT-associated migration [25]. The results of this study revealed that nuclear accumulation of Slug and Twist was inversely correlated with membranous E-cadherin expression $(p=0.001 ; p<0.001)$ and up-regulation of Vimentin expression $(p<0.001 ; p=0.007)$. Based on the associations of loss of membranous E-cadherin and its cytoplasmic accumulation with nuclear Slug and Twist expression, we hypothesized that Slug and Twist are transcriptional suppressors of E-cadherin and inducers of Vimentin in ICONST. These relationships are more obvious in metaplastic carcinoma than in ICONST.

In the current study, expressions of E-cadherin and Vimentin were found to influence PFS and OS. The patients expressing E-cadherin had better outcomes $(p=0.04)$. Conversely, increased Vimentin expression was associated with a poorer prognosis $(p=0.042)$, consistent with the findings of a previous study conducted by Kokkinos et al. [37]. These results confirm the negative impacts of EMT on the prognosis of TNBC. EMT markers were highly expressed in the SpCC and MPC tumors in our study. Similar results have been reported by Hennessy et al. [39]. These findings might at least partly explain the poor prognoses of these two histological subtypes, as speculated in a previous study [39]. In our study, the patients with positive Slug and Twist expression had poor PFS and OS, although these findings were not significant. Furthermore, tumor size $(p=0.036)$, TNM stage $(p=0.001,<0.001)$ and E-cadherin $(p=0.04)$ were identified as independent prognostic factors for TNBC in analyses using data adjusted for all clinicopathological parameters. We will conduct further research for the role of EMT in the prognosis of TNBC patients.

Chemotherapy is the mainstay of systemic treatment for patients with TNBC at all disease stages.

\section{Abbreviation}

AJCC: American Joint Committee on Cancer; EMT: epithelial-to-mesenchymal transition; ER: estrogen receptor; ICONSTs: invasive carcinomas of no special type; HER2: human epidermal growth factor receptor 2; MPCs: matrix-producing carcinomas; OS: overall survival; PR: progesterone receptor; PFS: progression-free survival; SpCCs: spindle cell carcinomas; TN: triple-negative; TNBC: triplenegative breast cancer.
The lack of targeted therapies and the poor prognosis of these patients have prompted a major effort to discover actionable molecular targets for the treatment of patients with these tumors. In addition, identification of groups of patients who are candidates for specific treatment regimens continues to represent another major research issue. Based on the TNBC classification proposed by Lehmann et al. [5], the 7 subtypes have very different predicted pathological complete response ( $\mathrm{pCR}$ ) rates (high vs. low) to current standard chemotherapy regimens [43]. These different subtypes have been reported to be closely correlated with several specific histological subtypes of TNBC [5]. The recognition of certain specific subtypes of TNBC might allow for the identification of women with extremely good or extremely poor prognoses, which would facilitate the selection of appropriate treatments for these patients. Emerging evidence suggests that molecular and phenotypic associations exist between the EMT and chemoresistance in several cancers [44,45], and such associations might be responsible for the resistance of metaplastic TNBC to cytotoxic agents [16]. Specially, late-stage SpCC and MPC, which are characterized by high expression of EMT markers and poor prognosis, should be treated using more active and effective agents. The molecular differences among the specific TNBC subtypes may translate into the use of different therapeutic approaches. In recent years, a number of studies have focused on the identification of molecules that might be effectively targeted in TNBC, including targets of EMT regulation [36,46]. Our findings provide a theoretical basis for the development of treatments targeting the EMT in TNBC. Furthermore, patients with SpCC and MPC would gain much more clinical benefit from EMT-targeted regimens. Therefore, the SpCC and MPC subtypes might be the best candidates for targeted therapies based on alterations in the EMT and stem cell markers. However, these subtypes of TNBC are rare tumors with extremely low incidences. We realized that the sample size of SpCC and MPC in this study was relatively small. Further studies in a larger cohort are needed to validate our findings.

\section{Supplementary Material}

Supplementary tables.

http://www.jcancer.org/v09p0604s1.pdf

\section{Ethical Approval}

Experiments and data generation were in accordance with the ethical standards of relevant national and international rules and regulations (GCP, Declaration of Helsinki). 


\section{Competing Interests}

The authors have declared that no competing interest exists.

\section{References}

1. Perou CM, Sørlie T, Eisen MB, et al. Molecular portraits of human breast tumours. Nature. 2000; 406: 747-52. doi: 10.1038/35021093.

2. Prat A, Ellis MJ, Perou CM. Practical implications of gene-expression-based assays for breast oncologists. Nat Rev Clin Oncol. 2011; 9: 48-57. doi: 10.1038/nrclinonc.2011.178

3. Prat A, Perou CM. Deconstructing the molecular portraits of breast cancer. Mol Oncol. 2011; 5: 5-23. doi: 10.1016/j.molonc.2010.11.003.

4. Sørlie T, Perou CM, Tibshirani R, et al. Gene expression patterns of breast carcinomas distinguish tumor subclasses with clinical implications. Proc Natl Acad Sci USA. 2001; 98: 10869-74. doi: 10.1073/pnas.191367098.

5. Lehmann BD, Bauer JA, Chen X, et al. Identification of human triple-negative breast cancer subtypes and preclinical models for selection of targeted therapies. J Clin Invest. 2011; 121: 2750-67. doi: 10.1172/JCI45014.

6. Arias AM. Epithelial mesenchymal interactions in cancer and development. Cell. 2001; 105: 425-31. doi: 10.1016/S0092-8674(01)00365-8.

7. Montserrat N, Gallardo A, Escuin D, et al. Repression of E-cadherin by SNAIL, ZEB1, and TWIST in invasive ductal carcinomas of the breast: a cooperative effort? Hum Pathol. 2011; 42: 103-10. doi: 10.1016/j.humpath.2010.05.019.

8. Pomp V, Leo C, Mauracher A, et al. Differential expression of epithelial-mesenchymal transition and stem cell markers in intrinsic subtypes of breast cancer. Breast Cancer Res Treat. 2015; 154: 45-55. doi: $10.1007 /$ s10549-015-3598-6

9. Hammond MEH, Hayes DF, Dowsett M, et al. American Society of Clinical Oncology/College of American Pathologists guideline recommendations for immunohistochemical testing of estrogen and progesterone receptors in breast cancer. J Clin Oncol. 2010; 28: 2784-95. doi: 10.1200/JCO.2009.25.6529.

10. Hammond ME, Hayes DF, Wolff AC. Clinical notice for American Society of Clinical Oncology-College of American Pathologists guideline recommendations on ER/PgR and HER2 testing in breast cancer. J Clin Oncol. 2011; 29: e458. doi: 10.1200/JCO.2011.35.2245.

11. Edge SB, Compton CC. The American Joint Committee on Cancer: The 7th edition of the AJCC cancer staging manual and the future of TNM. Ann Surg Oncol. 2010; 17: 1471-4. doi: 10.1245/s10434-010-0985-4

12. Page DL. Special types of invasive breast cancer, with clinical implications. Am J Surg Pathol. 2003; 27: 832-5. doi: 10.1097/00000478-200306000-00016.

13. Prasad CP, Rath G, Mathur S, et al. Expression analysis of E-cadherin, slug and GSK3beta in invasive ductal carcinoma of breast. BMC Cancer. 2009; 9: 325. doi: 10.1186/1471-2407-9-325.

14. Azoulay S, Laé M, Fréneaux P, et al. KIT is highly expressed in adenoid cystic carcinoma of the breast, a basal-like carcinoma associated with a favorable outcome. Mod Pathol. 2005; 18: 1623-31. doi: 10.1038/modpathol.3800483.

15. Colleoni M, Rotmensz N, Maisonneuve P, et al. Outcome of special types of luminal breast cancer. Ann Oncol. 2012; 23: 1428-36. doi: 10.1093/annonc/mdr461.

16. Hennessy BT, Giordano S, Broglio K, et al. Biphasic metaplastic sarcomatoid carcinoma of the breast. Ann Oncol. 2006; 17: 605-13. doi: 10.1093/annonc/mdlo06.

17. Montagna E, Maisonneuve P, Rotmensz N, et al. Heterogeneity of triple-negative breast cancer: histologic subtyping to inform the outcome. Clin Breast Cancer. 2013; 13: 31-9. doi: 10.1016/j.clbc.2012.09.002

18. Downs-Kelly E, Nayeemuddin KM, Albarracin C, et al. Matrix-producing carcinoma of the breast: an aggressive subtype of metaplastic carcinoma. Am J Surg Pathol. 2009; 33: 534-41. doi: 10.1097/PAS.0b013e31818ab26e.

19. Johnykutty S, Hicks D, Skinner K, et al. Spindle cell (sarcomatous) carcinoma of the breast: immunohistochemical profile and the clinical outcome. Lab Invest. 2009; 89: 48A-9A.

20. Moten AS, Jayarajan SN, Willis AI. Spindle cell carcinoma of the breast: a comprehensive analysis. Am J Surg. 2016; 211: 716-21. doi: 10.1016/j.amjsurg.2015.11.023.

21. Gersell DJ, Katzenstein AL. Spindle cell carcinoma of the breast. A clinocopathologic and ultrastructural study. Hum Pathol. 1981; 12: 550-61. doi: 10.1016/S0046-8177(81)80069-X

22. Irshad S, Ellis P, Tutt A. Molecular heterogeneity of triple-negative breast cancer and its clinical implications. Curr Opin Oncol. 2011; 23: 566-77. doi: 10.1097/CCO.0b013e32834bf8ae.

23. Micalizzi DS, Farabaugh SM, Ford HL. Epithelial-mesenchymal transition in cancer: parallels between normal development and tumor progression. J Mammary Gland Biol Neoplasia. 2010; 15: 117-34. doi: 10.1007/s10911-010-9178-9.

24. Martin TA, Goyal A, Watkins G, et al. Expression of the transcription factors snail, slug, and twist and their clinical significance in human breast cancer. Ann Surg Oncol. 2005; 12: 488-96. doi: 10.1245/ASO.2005.04.010.

25. Vuoriluoto K, Haugen $\mathrm{H}$, Kiviluoto S, et al. Vimentin regulates EMT induction by slug and oncogenic H-Ras and migration by governing Axl expression in breast cancer. Oncogene. 2011; 30: 1436-48. doi: 10.1038/onc.2010.509.
26. Usami $Y$, Satake $S$, Nakayama $F$, et al Snail-associated epithelial-mesenchymal transition promotes oesophageal squamous cell carcinoma motility and progression. J Pathol. 2008; 215: 330-9. doi: 10.1002/path.2365

27. Vergara D, Merlot B, Lucot JP, et al. Epithelial-mesenchymal transition in ovarian cancer. Cancer Lett. 2010; 291: 59-66. doi: 10.1016/j.canlet.2009.09.017.

28. Brabletz T, Hlubek F, Spaderna S, et al. Invasion and metastasis in colorectal cancer: epithelial-mesenchymal transition, mesenchymal-epithelial transition, stem cells and beta-catenin. Cells Tissues Organs. 2005; 179: 56-65. doi: $10.1159 / 000084509$.

29. Tsui KH, Lin YH, Chung LC, et al. Prostate-derived ets factor represses tumorigenesis and modulates epithelial-to-mesenchymal transition in bladder carcinoma cells. Cancer Lett. 2016; 375: 142-51. doi: 10.1016/j.canlet.2016.02.056

30. Mao $\mathrm{Y}, \mathrm{Zhang} \mathrm{N}, \mathrm{Xu}$ J, et al. Significance of heterogeneous Twist2 expression in human breast cancers. PLOS ONE. 2012; 7: e48178. doi: 10.1371/journal.pone.0048178.

31. Trimboli AJ, Fukino $\mathrm{K}$, de Bruin $\mathrm{A}$, et al. Direct evidence for epithelial-mesenchymal transitions in breast cancer. Cancer Res. 2008; 68: 937-45. doi: 10.1158/0008-5472.CAN-07-2148.

32. Asgeirsson KS, Jónasson JG, Tryggvadóttir L, et al. Altered expression of E-cadherin in breast cancer. Patterns, mechanisms and clinical significance. Eur J Cancer. 2000; 36: 1098-106. doi: 10.1016/S0959-8049(00)00062-9.

33. Jeong H, Ryu YJ, An J, et al. Epithelial-mesenchymal transition in breast cancer correlates with high histological grade and triple-negative phenotype. Histopathology. 2012; 60: E87-E95. doi: 10.1111/j.1365-2559.2012.04195.x.

34. Bergamaschi A, Kim YH, Kwei KA, et al. CAMK1D amplification implicated in epithelial-mesenchymal transition in basal-like breast cancer. Mol Oncol. 2008; 2: 327-39. doi: 10.1016/j.molonc.2008.09.004

35. Sarrió D, Rodriguez-Pinilla SM, Hardisson D, et al. Epithelial-mesenchymal transition in breast cancer relates to the basal-like phenotype. Cancer Res. 2008; 68: 989-97. doi: 10.1158/0008-5472.CAN-07-2017.

36. Al Moustafa AE. Epithelial-mesenchymal transition and its regulators are major targets of triple-negative breast cancer. Cell Adh Migr. 2013; 7: 424-5. doi: $10.4161 /$ cam. 26728 .

37. Kokkinos MI, Wafai R, Wong MK, et al. Vimentin and epithelial-mesenchymal transition in human breast cancer--observations in vitro and in vivo. Cells Tissues Organs. 2007; 185: 191-203. doi: 10.1159/000101320.

38. Gilles C, Thompson EW. The epithelial to mesenchymal transition and metastatic progression in carcinoma. Breast J. 1996; 2: 83-96. doi: 10.1111/j.1524-4741.1996.tb00076.x.

39. Hennessy BT, Gonzalez-Angulo AM, Stemke-Hale K, et al. Characterization of a naturally occurring breast cancer subset enriched in epithelial-to-mesenchymal transition and stem cell characteristics. Cancer Res. 2009; 69: 4116-24. doi: 10.1158/0008-5472.CAN-08-3441.

40. Cimino-Mathews A, Verma S, Figueroa-Magalhaes $\mathrm{MC}$, et al. A clinicopathologic analysis of 45 patients with metaplastic breast carcinoma. Am J Clin Pathol. 2016; 145: 365-72. doi: 10.1093/ajcp/aqv097.

41. Hajra KM, Chen DY, Fearon ER. The SLUG zinc-finger protein represses E-cadherin in breast cancer. Cancer Res. 2002; 62: 1613-8.

42. Yang J, Mani SA, Donaher JL, et al. Twist, a master regulator of morphogenesis, plays an essential role in tumor metastasis. Cell. 2004; 117: 927-39. doi: 10.1016/j.cell.2004.06.006.

43. Masuda H, Baggerly KA, Wang Y, et al. Differential response to neoadjuvant chemotherapy among 7 triple-negative breast cancer molecular subtypes. Clin Cancer Res. 2013; 19: 5533-40. doi: 10.1158/1078-0432.CCR-13-0799.

44. Creedon H, Gómez-Cuadrado L, Tarnauskaitè Ž, et al. Identification of novel pathways linking epithelial-to-mesenchymal transition with resistance to HER2-targeted therapy. Oncotarget. 2016; 7: 11539-52. doi: 10.18632/oncotarget.7317.

45. Fischer KR, Durrans A, Lee S, et al. Epithelial-to-mesenchymal transition is not required for lung metastasis but contributes to chemoresistance. Nature. 2015; 527: 472-6. doi: 10.1038/nature15748.

46. Ito K, Park SH, Nayak A, et al. PTK6 inhibition suppresses metastases of triple-negative breast cancer via SNAIL-Dependent E-cadherin regulation. Cancer Res. 2016; 76: 4406-17. doi: 10.1158/0008-5472.CAN-15-3445. 